\title{
Foot-ankle simulators: a tool to advance biomechanical understanding of a complex anatomical structure
}

\author{
Natsakis $\mathrm{T}^{* 1}$, Burg $\mathrm{J}^{1,2}$, Dereymaeker $\mathrm{G}^{1}$, Jonkers $\mathrm{I}^{2}$ and Vander \\ Sloten $J^{1}$ \\ ${ }^{1}$ Department of Mechanical Engineering, KU Leuven, Belgium \\ ${ }^{2}$ Department of Kinesiology and Rehabilitation Science, KU \\ Leuven, Belgium
}

August 26, 2015

\begin{abstract}
In vitro gait simulations have been available to researchers for more than two decades and have become an invaluable tool for understanding fundamental foot-ankle biomechanics. This has been realized through several incremental technological and methodological developments, such as the actuation of muscle tendons, the increase of controlled degrees of freedom and the use of advanced control schemes. Furthermore, in vitro
\end{abstract}

\footnotetext{
*Corresponding author:

Tassos Natsakis

Department of Mechanical Engineering, Biomechanics Section

Celestijnenlaan 300c, 3001 Heverlee, Belgium

e-mail: tassos.natsakis@kuleuven.be

tel: +3216372770
} 
experimentation enabled performing highly repeatable and controllable simulations of gait during simultaneous measurement of several biomechanical signals (e.g. bone kinematics, intra-articular pressure distribution, bone strain). Such signals cannot always be captured in detail using in vivo techniques, and the importance of in vitro experimentation is therefore highlighted. The information provided by in vitro gait simulations enabled researchers to answer numerous clinical questions related to pathology, injury and surgery. In this article, first an overview of the developments in design and methodology of the various foot-ankle simulators is presented. Furthermore, an overview of the conducted studies are outlined and an example of a study aiming at understanding the differences in kinematics of the hindfoot, ankle and subtalar joints after total Ankle Arthroplasty (TAA) is presented. Finally, the limitations and future perspectives of in vitro experimentation and in particular of foot-ankle gait simulators are discussed. It is expected that the biofidelic nature of the controllers will be improved in order to make them more subject specific and to link foot motion to the simulated behaviour of the entire missing body, providing additional information for understanding the complex anatomical structure of the foot.

Keywords - Foot-ankle biomechanics, in vitro gait simulations, surgery, pathology, injury

\section{Introduction}

The investigation and treatment of foot related pathologies requires thorough knowledge on the biomechanical behaviour of the individual structures of the foot, i.e the bones, ligaments, muscles, tendons and soft-tissue. Several methodologies have been developed over the years to deepen the biomechanical knowledge of the foot-ankle complex and hence to assist clinicians in better managing 
foot-related pathologies: Radiology allows clinicians to visualise the internal structures of the foot, mainly those of higher density i.e. the bones, by creating a two-dimensional imprint. Even though this often aids in identifying specific structural deformities and fractures, it does not provide information on the soft tissues surrounding the bones and it is generally limited to two-dimensional (2D) images. Three-dimensional (3D) imaging modalities such as computed tomography $(\mathrm{CT})$ and magnetic resonance imaging (MRI) techniques allow to study soft-tissues such as ligaments, cartilage, tendons etc., in a non-invasive manner ${ }^{1,2}$. In most cases, CT and MR images are captured in a lying and therefore non-weight bearing position. This reduces the relevance of the images for locomotion since weight bearing considerably changes the foot bone positions. Weight bearing acquisitions have the potential to reveal specific weight-bearing related pathologies (e.g. flatfoot deformity) ${ }^{3}$. However, radiography, CT and MRI are all static modalities, and cannot provide information on the functional, i.e. dynamic behaviour of the different foot structures.

To study dynamic foot function, several researchers used integrated 3D motion capture techniques. Using active or passive markers attached to the skin, the kinematics of foot segments, i.e. a functional grouping of different foot bones, can be calculated ${ }^{4,5}$. These measurements can be complemented by electromyographic (EMG) measurements that reveal the activation pattern of muscles of the lower leg $6,7,8$. However, this approach cannot document the kinematics of the individual bones, nor the actual force applied by the muscles. Furthermore, skin-motion artefacts limit the accuracy of the measured kinematics and for specific bones (e.g. talus) no markers can be placed to track their position. Furthermore, EMG measurements only provide a measure of the proportional activation of a specific muscle and do not provide information about the actual magnitude of the muscle force production. 
In recent years, biomedical engineering has focussed on substantiating insights gained from the descriptive studies on foot-related treatment strategies using biomechanical modelling. Using musculoskeletal models that represent the patient specific geometry of the bones, ligament and tendon attachment locations are represented and using dedicated modelling formulations (being either rigid body dynamics or finite element analysis or a combination of both), the response of the patient to a specific surgical intervention is calculated ${ }^{9,10}$. However, such models require thorough validation in terms of bone kinematics and joint contact forces given known motion and muscle actuation patterns. This level of validation cannot be provided through gait analysis and static medical imaging. Therefore, investigators have relied on in vitro investigation of the functional behaviour of the foot-ankle complex. Through in vitro experimentation, it is possible to use highly invasive techniques and assess the kinematic and kinetic behaviour of individual foot structures. In vitro experimentation provides a standardised test allowing to apply highly repeatable test protocols that reduce intra-specimen variability. This allows to study the consecutive effect of subsequent surgical interventions (e.g. prosthesis implantation, osteotomy etc.) in one and the same cadaveric specimen. As such the effect of different stages of a surgical intervention can be studied, while neglecting patient-specific compensation strategies. Even though in vitro experimentation offers many advantages, it is also bound to specific difficulties. Special apparatuses need to be designed that allow the simulation of functional behaviour of the foot-ankle complex comparable to physiological gait, or other types of locomotion. Ideally, these apparatuses should induce physiological foot-ankle motion by applying realistic loading profiles to the various tendons of the foot muscles. Several research groups have performed in-vitro experiments ranging from simple static measurements to fully automated gait simulations with muscle actuation. In this review 
article, the steps and developments that were taken over the past decades are first outlined in the following section. Then, the studies that have used in vitro simulators are presented in the next two sections. An example of an application from research from our own group is also present. Finally, a discussion is present outlining the main limitations and perspectives for the future of foot ankle simulator.

\section{State of the art on foot ankle simulators}

\section{Developments on the hardware design}

The first studies that automated the in vitro study of foot-ankle biomechanics, were of static nature: the foot sole was compressed against the fixated tibia un-

der varying levels of loads ${ }^{11,12,13,14}$, allowing to study various intrinsic signals under physiologic foot compression. This methodology, although innovative for that time, provided information only in a specific static position of the foot, which might not be representative for the gait cycle. This was both due to the design of the experimental set-up but also due to limitations of the available measurement technique. New experimental set-ups concentrated on simulating the ankle motion along different physiological degrees of freedom (DoF): Vrahas et al. ${ }^{15}$ was the first to simulate plantar-dorsiflexion motion whereas Calhoun et al. ${ }^{16}$ and Hintermann et al. ${ }^{17}$ imposed plantar-dorsiflexion and in-eversion under varying loading levels. In all three studies, the foot was positioned on top of a plate with variable position and different levels of compressive loading were applied to the tibia, using an electric or a pneumatic actuator. These set-ups therefore allowed to study foot biomechanics not only under varying level of external loading, but also relative to the foot position representative for specific phases of the gait cycle. Rosenbaum et al. ${ }^{18}$ improved upon the existing designs 
by applying static load to the tendons of three extrinsic muscles of the lower leg (Achilles tendon, flexor digitorum longus and flexor hallucis longus) using pulleys and weights, being the first set-up to document the effect of muscle actuation on foot biomechanics.

Even though these static and quasi-static set-ups revealed important aspects on foot-ankle biomechanics, the need for a more dynamic set-up allowing to impose motion dynamically was apparent. The study of Sharkey and Hamel ${ }^{19}$, was the first to present a dynamic cadaveric set-up. The set-up simulated a roll-off in 11 seconds driving the horizontal kinematics and manipulating the vertical kinematics using two guiding rails with a specific profile. The rotational kinematics in the sagittal plane were enforced by the friction between a constraining platform and the specimens. Muscle forces were applied dynamically on five tendons (Triceps suraey, tibialis posterior, peroneal muscles combined, flexor hallucis longus and flexor digitorum longus) using independently controlled linear actuators. This set-up therefore included all the essential elements of a realistic, dynamic gait simulation.

Although this set-up was a significant breakthrough for in-vitro research on the foot-ankle complex, it was an over-constrained design that lacked the flexibility of applying scaled kinematics depending on the specific dimensions of the specimens. To address this issue, Kim et al. ${ }^{20}$ and Hurschler et al. ${ }^{21}$ introduced simulators that allowed full control of the sagittal plane kinematics. This simplification is a valid design restriction as the main components of the foot-ankle kinematics during gait relate to the sagittal plane. In Kim et al. ${ }^{20}$, the foot was mounted on a carriage, able to translate in the antero/posterior and proximal/distal directions and to rotate in the sagittal plane. Six actuators were mounted on the carriage and attached to muscle tendons simulating muscle action. One roll-off was performed in 5 seconds. Hurschler et al. ${ }^{21}$ followed a 
different approach in their design: the foot was fixed proximally and a plate beneath the foot simulated the sagittal plane kinematics. Seven muscle actuators were attached to nine tendons of the foot (Achilles tendon, tibialis posterior, flexor hallucis longus, flexor digitorum longus, peronei, tibialis anterior, extensor digitorum longus and extensor hallucis longus), simplifying the design. A roll-off was performed in 60 seconds and a varying muscle force pattern was applied. Besides the increase of the controlled degrees of freedom that these designs brought, there was also an increase in the number of simulated muscle forces (6 and 7 muscles respectively).

The next generation of gait simulators further increased the number of controlled degrees of freedom (DoF), with a full 6-DoFs simulation realized by Aubin et al. ${ }^{22}$ and Noble et al. ${ }^{23}$. Both of these systems featured a platform that simulated kinematics in 6 degrees of freedom, with the specimen fixed against a static frame. All the muscle control units were also fixed to the static frame, similar to the approach of Hurschler et al. ${ }^{21}$. Besides the increase of simulated DoFs, there was also a considerable increase in the simulation speed: Aubin et al. ${ }^{22}$ reported simulations at a nearly physiologic speed of $1.5 \mathrm{sec}$ per roll-off, using a prosthetic foot, whereas Noble et al. ${ }^{23}$ reported a roll-off of 3.2 seconds. Finally, a new design was also introduced by Peeters et al. ${ }^{24}$, simulating kinematics in 3 DoF (anterior/posterior and proximal/distal displacement and sagittal rotation) under simultaneous actuation of six tendons (tibialis anterior, tibialis posterior, peronei, Achilles tendon, flexor hallucis longus, flexor digitorum longus). This design was further developed by Natsakis et al. ${ }^{25}$ and allowed simulations with a roll-off speed of 1 second. 


\section{Advances in the control of gait simulators}

One of the major limitations of state of the art gait simulations relates to the choice of input signals. From the very first simulations, the imposed input kinematics and kinetics were derived directly from in vivo measurements of lower leg kinematics of control subjects. This approach, however, does not take into account differences in the biomechanical behaviour between specimens, as preferably different kinematics and kinetics should be used for the simulation of gait characteristics of specimens with different anthropometric dimensions ${ }^{19,26}$. To address this issue, the control schemes of gait simulators needed to be further developed. Aubin et al. ${ }^{22}$ implemented iterative learning control, to improve the reliability and repeatability of the experimental set-up: during each trial, the controller adapted its response based on information gathered during previous trials, being able to predict the dynamic behaviour of the system. As a result, the controller could account for differences in the specimen dimensions. The controller was further improved a few years later, using a fuzzy-logic controller for the applied ground reaction force ${ }^{27}$. This allowed to iteratively alter the tibial kinetics and the force applied to the Achilles tendon and improved the quality of the simulations. Besides the improvements in the controller, Ren et al. ${ }^{28}$ and Natsakis et al. ${ }^{29}$ defined kinematics models of the leg and foot to calculate specimen-specific kinematics based on anthropometric measurements. As a result, the specific input kinematics were calculated for each specimen and were shown to reproduce more accurate kinematic and kinetic conditions.

One of the distinctions between different gait simulator designs related to the specific signal used to control each degree of freedom. In all simulator designs, the degrees of freedom were position controlled, with the exception of the vertical translation degree of freedom. Whereas some of the groups were controlling the vertical kinematics ${ }^{19,22,23,24}$, others were controlling the vertical ground re- 
action force ${ }^{21,20}$. The initial simulations were performed with a position control for the vertical direction, causing much variability in the measured ground reaction force. This is due to the high sensitivity of the ground reaction force to small position errors during the simulations. This error is larger when dealing with specimens of different dimensions and when not using a specimen specific kinematics model. Therefore, many investigators performed studies that controlled the vertical ground reaction force instead of the vertical kinematics. Even though the control of the vertical ground reaction force significantly improved the quality of the simulations, it was still an over-constrained model: All degrees of freedom were controlled and it lacked therefore a DoF that could adapt and reflect the changes in the physiologic conditions of the cadaveric specimens e.g. in post-surgical conditions. This is important, as altering the physiological condition of the specimen (e.g. after a surgery), is expected to affect both the ground reaction forces and the kinematics ${ }^{30,31}$. Indeed, when performing new types of surgery, ground reaction forces or kinematics cannot be obtained from a patient population and no information is available on the form of these signals during stance phase. To address this issue and to allow performing physiological gait simulations in specimens after surgery, our group developed a new methodology called the inertial controller ${ }^{25}$. This technique allowed simulations of gait without pre-knowledge on the vertical kinematics or ground reaction force conditions by simulating the effects of gravity and inertia during locomotion. 


\section{Research questions addressed through in vitro ex- perimentation}

The first signals measured during in vitro experimentation of the foot were related to the contact characteristics of the ankle joint. This was at least partially driven by the availability of pressure sensitive FujiFilm, a new method for measuring contact characteristics in synovial joints ${ }^{32,33}$. The pressure sensitive film was inserted in the ankle and subtalar joint. During compression of the foot sole to the fixated tibia, the contact area and distribution were documented ${ }^{11,12,13}$. This allowed testing the hypothesis that cartilage pathology relates to high contact stress. Vrahas et al. ${ }^{15}$ and Calhoun et al. ${ }^{16}$ also measured loading conditions in the ankle joint using a pressure sensitive film. Both set-ups allowed to relate joint loading conditions not only to the level of external loading, but also to the foot position representative for specific phases of the gait cycle. With the development of dynamic gait simulators, joint loading conditions, and more specifically intra articular pressure distribution, were measured also dynamically, mainly in the ankle joint ${ }^{34,35,36,37,38,39,40,41}$ but also the chopart joint ${ }^{42}$, the subtalar joint ${ }^{43}$, and four midfoot joints ${ }^{44}$. The availability of thin, flexible and dynamic pressure sensors, mainly based on piezoresistive transducers, was crucial in this respect.

Bone strains were measured during dynamic simulations, in an attempt to validate finite element models ${ }^{45}$. Using strain gauges, strain measurements were

performed at the second and fifth metatarsals. Niu et al. ${ }^{46}$ later measured bone strains of the navicular and medial cuneiform under static compression and Achilles tendon actuation. In addition plantar pressure distribution was measured during dynamic gait simulations, first by Hamel et al. ${ }^{47}$ and later on by Kim et al. ${ }^{48}$, Wulker et al. ${ }^{49}$ and Edwards et al. ${ }^{50}$. The plantar pressure was, 
and still is, used extensively as a quality control and validation of the reference gait simulations, as its pattern is well documented and rather uniform in control subjects.

To further unlock the potential of dynamic gait simulations, many groups focused on more intrinsic signals such as specific bone kinematics. Indeed, the difficulty to directly measure bone kinematics during in-vivo measurements, hindered the development of accurate musculoskeletal models. Kitaoka et al. ${ }^{14}$ was the first to measure the position of individual bones (talus, navicular, calcaneus and first metatarsal) using magnetic sensors. The relative position of the bones was documented in a static position of the foot under varying loads of $0 \mathrm{~N}, 222 \mathrm{~N}, 445 \mathrm{~N}$ and $667 \mathrm{~N}$ compression of the tibia against the sole of the foot. Many groups documented foot bone kinematics during dynamic simulations of gait, starting with Hamel et al. ${ }^{51}$ (documenting tibia, talus, calcaneus) followed by Nester et al. ${ }^{52,53}$ (documenting tibia, talus, calcaneus, navicular, cuneiforms, cuboid, metatarsals 1, 2, 3, 4 and 5): Whittaker et al. ${ }^{26}$, Aubin et al. ${ }^{54}$ (documenting tibia, talus, calcaneus, navicular, medial cuneiform, cuboid, metatarsals 1, 3 and 5): Peeters et al. ${ }^{24}$, Burg et al. ${ }^{55}$, Okita et al. ${ }^{56}$ (documenting tibia, talus, calcaneus, navicular, cuboid).

Apart from insights related to the native foot, gait simulations were used to contrast the native and pathologic or surgically induced conditions in the same specimens. This is the main advantage of in vitro experimentation as they allow to study the exact same specimen in two different conditions and to isolate the effect of the pathology/surgery on the various outcome measures.

Konradsen et al. ${ }^{57}$ studied the risk of ankle sprains using a custom-built apparatus capable of simulating swing and heel strike phases of the gait cycle in cadaveric specimens. By altering the inclination of the ground during the initial contact, they could investigate the inclinations that caused ankle sprains and 
therefore predict the occurrence of sprains during normal gait. Lee et al. ${ }^{44}$ studied the diabetic foot and its effect on the joint contact pressure of the midfoot joints. They performed gait simulations using the experimental set-up described by Noble et al. ${ }^{23}$ using a control $(\mathrm{N}=8)$ and a diabetic $(\mathrm{N}=8)$ group. The intraarticular pressure in the four joints of the midfoot was found to be statistically increased in the diabetic feet, compared to the native ones. Finally, flat-foot deformity was studied by Jackson et al. ${ }^{58}$ and Watanabe et al. ${ }^{59}$. In both studies, normal feet were used to simulate stance phase before and after simulating flat-foot deformity by surgically attenuating specific ligaments (i.e spring, talocalcaneal interosseous, superficial deltoid and plantar naviculocuneiform ligaments). As the imposed kinematics and muscle forces were identical before and after the surgical intervention, the changes in the measured kinematics reflected the isolated effect of the specific ligament and its contribution to flat foot deformity. Besides studying pathology, gait simulations are ideal to investigate the effect of surgery. Valderrabano et al. ${ }^{60}$ compared the range of motion (ROM) of several joints in cadaveric ankles under three conditions: 1) native, 2) after ankle arthrodesis and 3) after TAA. Their experimental set-up allowed applying a maximum moment to each of the three rotational axes of the ankle, after which the relative position of the bones was measured using reflective markers. Finally, Nicholson et al. ${ }^{61}$ and Fukuda et al. ${ }^{62}$ studied the effect of misalignment of the two components of the Agility TAA (Depuy, Warsaw, IN). They were able to demonstrate the negative effects of this misalignment on the wear of the prosthetic components, quantifying the intra-articular pressure distribution using piezoresistive pressure sensing arrays.

In addition, other surgical techniques have been studied using in vitro gait simulations; Bayomy et al. ${ }^{63}$ studied the effect of the first metatarsophalangeal joint arthrodesis on the plantar pressure distribution, while Van Bergen et al. ${ }^{39}$ and 
Anderson et al. ${ }^{64}$ focused on talar dome resurfacing and its influence on joint loading conditions. Finally, Trask et al. ${ }^{65}$ investigated the effect of osteotomy of the second metatarsal on plantar pressure peak, whereas Meardon et al. ${ }^{66}$ studied the effect of orthotics on the ground reaction forces and on the bone strain of the metatarsal bone. In all of these studies, the use of gait simulations allowed to explore the effect of each specific intervention, without any harmful effects on patients.

Our group used a custom-built cadaveric gait simulator ${ }^{24}$ to investigate the effect of total ankle prosthesis (TAP) on the kinematics of the ankle and subtalar joints. In this study, nine freshly frozen cadaveric specimens were used to perform gait simulations before and after the implantation of a three components Hintegra TAP (New deal, Lyon, France). The simulations were performed using a specimen specific kinematics model Natsakis et al. ${ }^{29}$, which determined the input kinematics for the horizontal and sagittal rotation kinematics of the simulation, whereas the inertial controller Natsakis et al. ${ }^{25}$, determined the ground reaction force and kinematics in the vertical direction. Each simulated roll-off lasted 1 second and five repetitions were performed for each specimen and each case. The position of five bones of the hindfoot (tibia, talus, and calcaneus) was determined using a cluster of active markers mounted on top of bone pins that were previously inserted and fixated on the bones. The position of the markers was captured using a Krypton Optoelectronic Motion Capture System (Krypton $\mathrm{K} 600$, Metris, Belgium) at $100 \mathrm{~Hz}$ and the relative position between the bones was calculated by constructing virtual coordinate frames on each bone (Figure 2). By calculating the joint kinematics in the three anatomical planes (Sagittal, Coronal, Transverse), the total range of motion (ROM) for each bone combination was determined during Initial Double Support (IDS), Single Support (SS) and Terminal Double Support (TDS) phases of stance. The ROM 
prior and after TAP was compared for each joint demonstrating the influence of the TAP on the ankle and subtalar kinematics. The results suggest that even though there is little influence of the TAP on the overall hindfoot kinematics, several differences are seen in the ankle and subtalar joints (Figure 3). Especially in the coronal plane, where the TAP does not influence hindfoot motion, ankle ROM increases on average with three degrees whereas the subtalar ROM decreases on average with two degrees during SS phase. This shift in ROM between ankle and subtalar joint can only be documented through in vitro experimentation.

\section{Discussion}

From the very early investigations of foot-ankle kinematics by Isman et al. ${ }^{67}$ until today, in vitro simulators with increasing complexity were developed to study the dynamic function of the foot-ankle structure. History has shown that in vitro simulations, when properly designed, are complementary to in vivo studies. Several research groups have contributed in achieving dynamic gait simulations featuring six controlled degrees of freedom, multiple muscle actuation, specimen and condition specificity and roll-offs at near physiologic speed. In vitro simulators allow clinicians and engineers to investigate foot function in pathology and after surgical intervention in a highly invasive and repeatable way. The bone kinematics, joint loading conditions, bone or ligament strains can be measured in specimens before and after simulating a specific pathology or performing a surgical intervention. The imposed kinematics and muscle forces can be controlled and altered separately, reflecting their individual effect on the measured signals.

Even though in vitro gait simulations allow to gain important insights on footankle function, this approach is not without its limitations. Firstly, the soft 
tissues of the cadaveric specimens undergo certain deterioration over time, and therefore changes in material properties (e.g. stiffness, wear etc.) might affect their functional behaviour. The advances in automation to increase the simulation speed aim at reducing the influence of this limitation by lowering the time that each specimen is used. Secondly, all aforementioned studies perform a scaling on the forces applied to the cadaveric specimens, by reducing them commonly to $50 \%$ of BW. This will influence the measured outcome parameters during the simulations. However, reports in literature ${ }^{54}$ have demonstrated that such scaling has negligible influence on the resulting bone kinematics. Thirdly, cadaveric studies usually involve small number of subjects, hindering strong conclusions given the lack of extensive statistical testing. However, the increased repeatability of the in vitro simulations, amongst other due to improved control, inherently decreases the variability and errors during the measurements. This has the potential to increase the statistical power of in vitro simulation studies regardless of the small amount of specimens used.

Many studies have demonstrated the potential of gait simulations in exploring foot-ankle biomechanics; however, there are still many aspects that can be improved and explored further. One way to improve the quality and accuracy of the simulations, could be the implementation of smarter types of controllers. The use of iterative learning control introduced by Aubin et al. ${ }^{22}$ and Noble et al. ${ }^{23}$ could be further improved using model prediction control, a control methodology that has been used extensively in other fields for decades ${ }^{68}$. This will be possible by introducing more complex models of the gait simulators and of the foot as pre-knowledge in the controller.

In addition, better ways to calculate and impose the muscle actuation during simulations can be found. Currently, most studies derive the muscle forces that are applied during the simulation through inverse dynamic musculoskeletal 
modelling. However, this modelling is subject specific and might therefore not necessarily correspond to each specific cadaveric specimen. Similar to the specimen specific kinematic model that has been developed ${ }^{29}$, a specimen specific muscle force model could be useful for performing more realistic and reliable simulations.

The signals measured during dynamic gait simulations, could also be extended to measure strain of ligaments or tendons, or deformation of soft tissue (e.g. heel pad, plantar aponeurosis etc.), as has been performed in simulations of the knee ${ }^{69}$. Such information could be crucial for construction of more accurate musculoskeletal and joint contact models. This requires integrating readily available strain gauges and ultrasound measurement devices in the current designs of gait simulators. Furthermore, currently only the loading conditions normal to the surface of the joints can be measured; shear loading however is of utmost importance when investigating joint disorders and cartilage wear during gait ${ }^{70}$. Therefore, developing new techniques for measuring the shear component of joint loading during gait can help in better understanding the development of osteoarthritis and prosthesis wear.

Finally, in-vitro dynamic simulations can be utilised to study other types of locomotion, besides normal gait. The cases of stair ascending-descending, squatting or even running could further aid the understanding of foot function. Other types of simulators, such as for instance for the knee ${ }^{71,72,73}$ or the hip ${ }^{74}$ have already attempted to simulate such types of locomotion.

In the past 20 years, several in vitro gait simulators have been developed to advance the understanding of human foot biomechanics. Review of the literature clearly indicates the added value of in vitro gait simulations. Given the recent methodological developments, their role in functional outcome evaluation of different surgical interventions and validation of biomechanical modelling tech- 
niques is only expected to become more important. By improving the biofidelic nature of the controllers in order to make them more subject specific and to link foot motion to the simulated behaviour of the entire missing body, it is expected to broaden the scope of these measurements and provide additional information for better understanding the complex anatomical structure of the foot.

\section{References}

[1] Kerr R, Forrester DM and Kingston S. Magnetic resonance imaging of foot and ankle trauma. Orthop Clin North Am 1990; 21: 591-601.

[2] Potter HG, Deland JT, Gusmer PB et al. Magnetic Resonance Imaging of the Lisfranc Ligament of the Foot. Foot Ankle Int 1998; 19: 438-446.

[3] Zhang $\mathrm{Y}, \mathrm{Xu}$ J, Wang $\mathrm{X}$ et al. An in vivo study of hindfoot 3D kinetics in stage II posterior tibial tendon dysfunction (PTTD) flatfoot based on weight-bearing CT scan. Bone Joint Res 2013; 2: 255-63.

[4] Lafortune M, Cavanagh P, Sommer III H et al. Three-dimensional kinematics of the human knee during walking. J Biomech 1992; 25: 347-357.

[5] Whittle M. Clinical gait analysis: A review. Hum Mov Sci 1996; 15: 369-387.

[6] Tomaro J and Burdett RG. The effects of foot orthotics on the EMG activity of selected leg muscles during gait. J Orthop Sports Phys Ther 1993; 18: 532-6.

[7] Bogey R, Perry J and Gitter A. An EMG-to-Force Processing Approach for Determining Ankle Muscle Forces During Normal Human Gait. IEEE Trans Neural Syst Rehabil Eng 2005; 13: 302-310. 
[8] Murley GS, Landorf KB, Menz HB et al. Effect of foot posture, foot orthoses and footwear on lower limb muscle activity during walking and running: a systematic review. Gait Posture 2009; 29: 172-87.

[9] Koopman B, Grootenboer HJ and de Jongh HJ. An inverse dynamics model for the analysis, reconstruction and prediction of bipedal walking. $J$ Biomech 1995; 28: 1369-1376.

[10] Haraguchi N, Armiger RS, Myerson MS et al. Prediction of threedimensional contact stress and ligament tension in the ankle during stance determined from computational modeling. Foot ankle Int 2009; 30: 177-85.

[11] Tarr RR, Resnick CT, Wagner KS et al. Changes in Tibiotalar Joint Contact Areas Following Experimentally Induced Tibial Angular Deformities. Clin Orthop Relat Res 1985; 199: 72-80.

[12] Macko VW, Matthews LS, Zwirkoski P et al. The joint-contact area of the ankle. The contribution of the posterior malleolus. J Bone Joint Surg Am 1991; 73: 347-51.

[13] Wagner UA, Sangeorzan BJ, Harrington RM et al. Contact characteristics of the subtalar joint: load distribution between the anterior and posterior facets. J Orthop Res 1992; 10: 535-43.

[14] Kitaoka HB, Lundberg a, Luo ZP et al. Kinematics of the normal arch of the foot and ankle under physiologic loading. Foot ankle Int / Am Orthop Foot Ankle Soc [and] Swiss Foot Ankle Soc 1995; 16: 492-499.

[15] Vrahas M, Fu F and Veenis B. Intraarticular contact stresses with simulated ankle malunions. J Orthop Trauma 1994; 8: 159-166.

[16] Calhoun JH, Li F, Ledbetter BR et al. A comprehensive study of pressure 
distribution in the ankle joint with inversion and eversion. Foot Ankle Int 1994; 15: 125-33.

[17] Hintermann B, Nigg BM, Sommer C et al. Transfer of movement between calcaneus and tibia in vitro. Clin Biomech 1994; 9: 349-355.

[18] Rosenbaum D, Bertsch C and Claes L. Tenodeses do not fully restore ankle joint loading characteristics: a biomechanical in vitro investigation in the hind foot. Clin Biomech 1997; 12: 202-209.

[19] Sharkey NA and Hamel AJ. A dynamic cadaver model of the stance phase of gait: performance characteristics and kinetic validation. Clin Biomech 1998; 13: 420-433.

[20] Kim KJ, Kitaoka HB, Luo ZP et al. In vitro simulation of the stance phase in human gait. J Musculoskelet Res 2001; 5: 113-121.

[21] Hurschler C, Emmerich J and Wülker N. In vitro simulation of stance phase gait part I: Model verification. Foot Ankle Int 2003; 24: 614-22.

[22] Aubin PM, Cowley M and Ledoux WR. Gait Simulation via a 6-DOF Parallel Robot With Iterative Learning Control. Biomed Eng IEEE Trans 2008; 55: 1237-1240.

[23] Noble LD, Colbrunn RW, Lee DG et al. Design and validation of a general purpose robotic testing system for musculoskeletal applications. J Biomech Eng 2010; 132: 025001-12.

[24] Peeters K, Natsakis T, Burg J et al. An in vitro approach to the evaluation of foot-ankle kinematics: performance evaluation of a custom-built gait simulator. Proc Inst Mech Eng H 2013; 227: 955-67.

[25] Natsakis T, Burg J, Dereymaeker G et al. Inertial control as novel technique for in vitro gait simulations. J Biomech 2015; 48: 392-395. 
[26] Whittaker EC, Aubin PM and Ledoux WR. Foot bone kinematics as measured in a cadaveric robotic gait simulator. Gait Posture 2011; 33: 645-650.

[27] Aubin PM, Whittaker E and Ledoux WR. A Robotic Cadaveric Gait Simulator With Fuzzy Logic Vertical Ground Reaction Force Control. IEEE Trans Robot 2012; 28: 246-255.

[28] Ren LL, Howard D, Nester CJ et al. A generic analytical foot rollover model for predicting translational ankle kinematics in gait simulation studies. $J$ Biomech 2010; 43: 194-202.

[29] Natsakis T, Peeters K, Burg F et al. Specimen-specific tibial kinematics model for in vitro gait simulations. Proc Inst Mech Eng Part H J Eng Med 2012; $227: 454-463$.

[30] Valderrabano V, Nigg BM, von Tscharner V et al. Gait analysis in ankle osteoarthritis and total ankle replacement. Clin Biomech (Bristol, Avon) 2007; 22: 894-904.

[31] Beischer aD, Brodsky JW, Polio FE et al. Functional Outcome and Gait Analysis After Triple or Double Arthrodesis. Foot Ankle Int 1999; 20: $545-553$.

[32] Daley RE, Engin AE and Gaughran GRL. Description of pressure sensitive paint transducer to measure joint contact forces. In Proc. 27th Annu. Conf. Eng. Med. Biol., vol. 16 (Alliance for Engineering in Medicine and Biology), 367.

[33] Ahmed AM. A pressure distribution transducer for in-vitro static measurements in synovial joints. J Biomech Eng 1983; 105: 309-314.

[34] Michelson JD, Checcone M, Kuhn T et al. Intra-articular load distribution in the human ankle joint during motion. Foot Ankle Int 2001; 22: 226-33. 
[35] Bertsch C, Rosenbaum D and Claes L. Intraartikuläre und plantare Druckverteilung des Sprung- gelenkkomplexes in Abhän- gigkeit von der Fußstellung *. Unfallchirurg 2001; 104: 426-433.

[36] McKinley TO, Rudert MJ, Koos DC et al. Contact stress transients during functional loading of ankle stepoff incongruities. J Biomech 2006; 39: 61726.

[37] Tochigi Y, Rudert MJ, Saltzman CL et al. Contribution of articular surface geometry to ankle stabilization. J Bone Joint Surg Am 2006; 88: 2704-13.

[38] Suckel A, Muller O, Wachter N et al. In vitro measurement of intraarticular pressure in the ankle joint. Knee Surgery, Sport Traumatol Arthrosc 2010; 18: $664-668$.

[39] van Bergen CJa, Zengerink M, Blankevoort L et al. Novel metallic implantation technique for osteochondral defects of the medial talar dome. A cadaver study. Acta Orthop 2010; 81: 495-502.

[40] Prisk VR, Imhauser CW, O'Loughlin PF et al. Lateral ligament repair and reconstruction restore neither contact mechanics of the ankle joint nor motion patterns of the hindfoot. J Bone Joint Surg Am 2010; 92: 2375-86.

[41] Natsakis T, Burg J, Dereymaeker G et al. Extrinsic Muscle Forces Affect Ankle Loading Before and After Total Ankle Arthroplasty. Clin Orthop Relat Res 2015; 473: 3028-3037.

[42] Suckel A, Muller O, Herberts $\mathrm{T}$ et al. Changes in Chopart joint load following tibiotalar arthrodesis: in vitro analysis of 8 cadaver specimens in a dynamic model. BMC Musculoskelet Disord 2007; 8: 80.

[43] Jung HG, Parks BG, Nguyen A et al. Effect of tibiotalar joint arthrodesis 
on adjacent tarsal joint pressure in a cadaver model. Foot ankle Int / Am Orthop Foot Ankle Soc [and] Swiss Foot Ankle Soc 2007; 28: 103-8.

[44] Lee DG and Davis BL. Assessment of the effects of diabetes on midfoot joint pressures using a robotic gait simulator. Foot ankle Int / Am Orthop Foot Ankle Soc [and] Swiss Foot Ankle Soc 2009; 30: 767-72.

[45] Donahue SW and Sharkey NA. Strains in the metatarsals during the stance phase of gait: implications for stress fractures. J Bone Joint Surg Am 1999; 81: $1236-1244$.

[46] Niu W, Tang T, Zhang M et al. An in vitro and finite element study of load redistribution in the midfoot. Sci China Life Sci 2014; 57: 1191-1196.

[47] Hamel aJ, Donahue SW and Sharkey NA. Contributions of active and passive toe flexion to forefoot loading. Clin Orthop Relat Res 2001; : 326334.

[48] Kim Kj, Uchiyama E, Kitaoka HB et al. An in vitro study of individual ankle muscle actions on the center of pressure. Gait Posture 2003; 17: $125-131$.

[49] Wülker N, Hurschler C and Emmerich J. In vitro simulation of stance phase gait part II: Simulated anterior tibial tendon dysfunction and potential compensation. Foot ankle Int / Am Orthop Foot Ankle Soc [and] Swiss Foot Ankle Soc 2003; 24: 623-629.

[50] Edwards WB, Ward ED and Derrick TR. Foot joint pressures during dynamic gait simulation. $J$ Foot Ankle Res 2008; 1: O21-O21.

[51] Hamel AJ, Sharkey NA, Buczek FL et al. Relative motions of the tibia, talus, and calcaneus during the stance phase of gait: a cadaver study. Gait Posture 2004; 20: 147-153. 
[52] Nester CJ, Jones RK, Liu A et al. Foot kinematics during walking measured using bone and surface mounted markers. J Biomech 2007; 40: 3412-3423.

[53] Nester CJ, Liu A, Ward E et al. Error in the description of foot kinematics due to violation of rigid body assumptions. J Biomech 2010; 43: 666-672.

[54] Aubin PM, Whittaker EC and Ledoux WR. Foot bone kinematics at half and three quarters body weight: A robotic cadaveric simulation of stance phase. In 2011 15th Int. Conf. Adv. Robot. (IEEE, Tallinn), 653-658.

[55] Burg J, Peeters K, Natsakis T et al. In vitro analysis of muscle activity illustrates mediolateral decoupling of hind and mid foot bone motion. Gait Posture 2013; 38: 56-61.

[56] Okita N, Meyers Sa, Challis JH et al. Midtarsal joint locking: new perspectives on an old paradigm. J Orthop Res 2014; 32: 110-5.

[57] Konradsen L and Voigt M. Inversion injury biomechanics in functional ankle instability: a cadaver study of simulated gait. Scand J Med Sci Sports 2002; 12: 329-336.

[58] Jackson LT, Aubin PM, Cowley MS et al. A robotic cadaveric flatfoot analysis of stance phase. J Biomech Eng 2011; 133: 051005.

[59] Watanabe K, Kitaoka HB, Fujii T et al. Posterior tibial tendon dysfunction and flatfoot: Analysis with simulated walking. Gait Posture 2013; 37: 264268.

[60] Valderrabano V, Hintermann B, Nigg BM et al. Kinematic changes after fusion and total replacement of the ankle: part 1: Range of motion. Foot Ankle Int 2003; 24: 881-7. 
[61] Nicholson JJ, Parks BG, Stroud CC et al. Joint Contact Characteristics in Agility Total Ankle Arthroplasty. Clin Orthop Relat Res 2004; 424: $125-129$.

[62] Fukuda T, Haddad SL, Ren Y et al. Impact of talar component rotation on contact pressure after total ankle arthroplasty: a cadaveric study. Foot ankle Int 2010; 31: 404-11.

[63] Bayomy AF, Aubin PM, Sangeorzan BJ et al. Arthrodesis of the First Metatarsophalangeal Joint: A Robotic Cadaver Study of the Dorsiflexion Angle. J Bone Jt Surg 2010; 92: 1754-1764.

[64] Anderson DD, Tochigi Y, Rudert MJ et al. Effect of implantation accuracy on ankle contact mechanics with a metallic focal resurfacing implant. $J$ Bone Joint Surg Am 2010; 92: 1490-500.

[65] Trask DJ, Ledoux WR, Whittaker EC et al. Second metatarsal osteotomies for metatarsalgia: a robotic cadaveric study of the effect of osteotomy plane and metatarsal shortening on plantar pressure. J Orthop Res 2014; 32: $385-93$.

[66] Meardon Sa, Edwards B, Ward E et al. Effects of custom and semi-custom foot orthotics on second metatarsal bone strain during dynamic gait simulation. Foot ankle Int / Am Orthop Foot Ankle Soc [and] Swiss Foot Ankle Soc 2009; 30: 998-1004.

[67] Isman RE, Inman VT and Poor PM. Anthropometric studies of the human foot and ankle. Bull Prosthet Res 1969; 11: 97-108.

[68] Morari M and H Lee J. Model predictive control: past, present and future. Comput Chem Eng 1999; 23: 667-682. 
[69] Delport H, Labey L, De Corte R et al. Collateral ligament strains during knee joint laxity evaluation before and after TKA. Clin Biomech 2013; 28: $777-782$.

[70] Setton La, Elliott DM and Mow VC. Altered mechanics of cartilage with osteoarthritis: Human osteoarthritis and an experimental model of joint degeneration. Osteoarthr Cartil 1999; 7: 2-14.

[71] Baldwin Ma, Clary C, Maletsky LP et al. Verification of predicted specimen-specific natural and implanted patellofemoral kinematics during simulated deep knee bend. J Biomech 2009; 42: 2341-8.

[72] Halloran JP, Clary CW, Maletsky LP et al. Verification of predicted knee replacement kinematics during simulated gait in the Kansas knee simulator. J Biomech Eng 2010; 132: 081010.

[73] Labey L, Innocenti B, Wong PD et al. Sensitivity of knee kinematics and soft tissues to quadriceps load near extension. J Orthop Transl Res Clin Appl 2011; 3: 27-37.

[74] Anderson AE, Ellis BJ, Maas SA et al. Validation of Finite Element Predictions of Cartilage Contact Pressure in the Human Hip Joint. J Biomech Eng 2008; 130: 1-10. 
Figures 


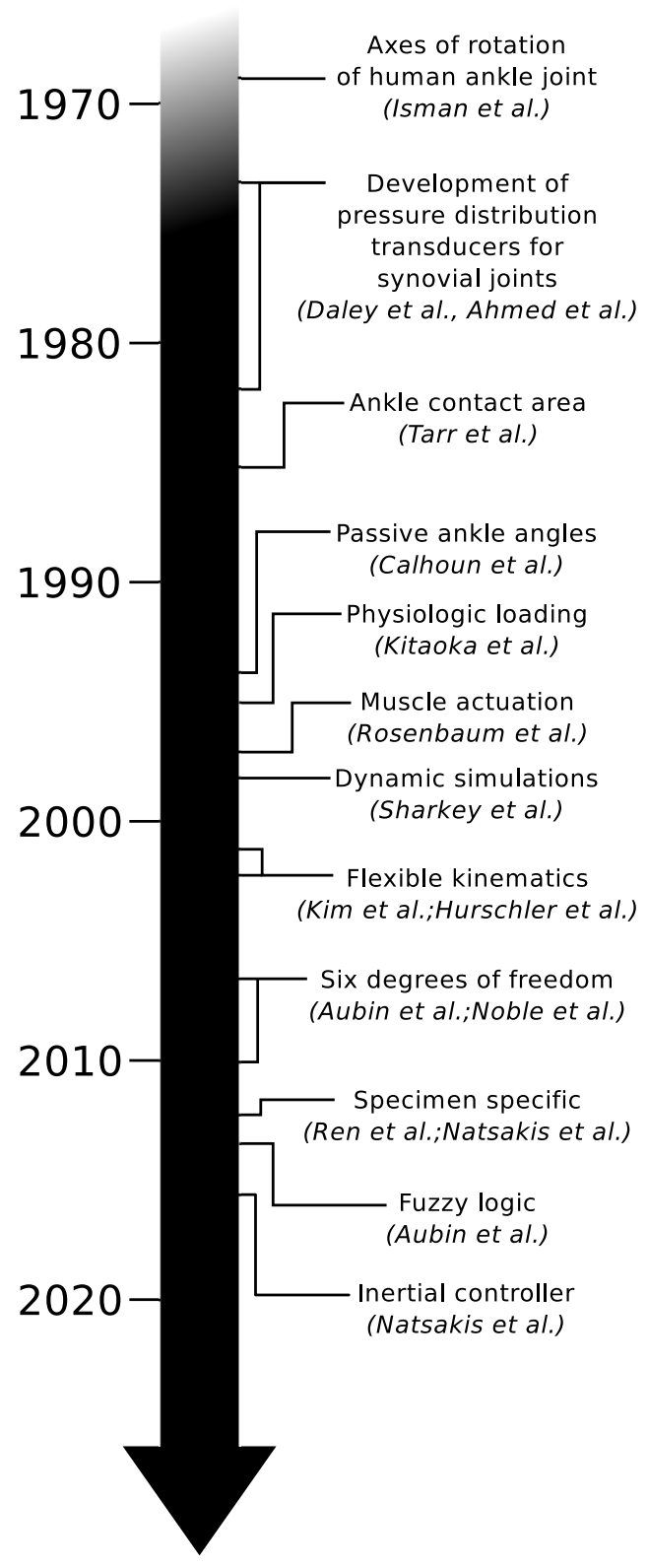

Figure 1: A time-line of major breakthroughs in in-vitro foot ankle simulators. 


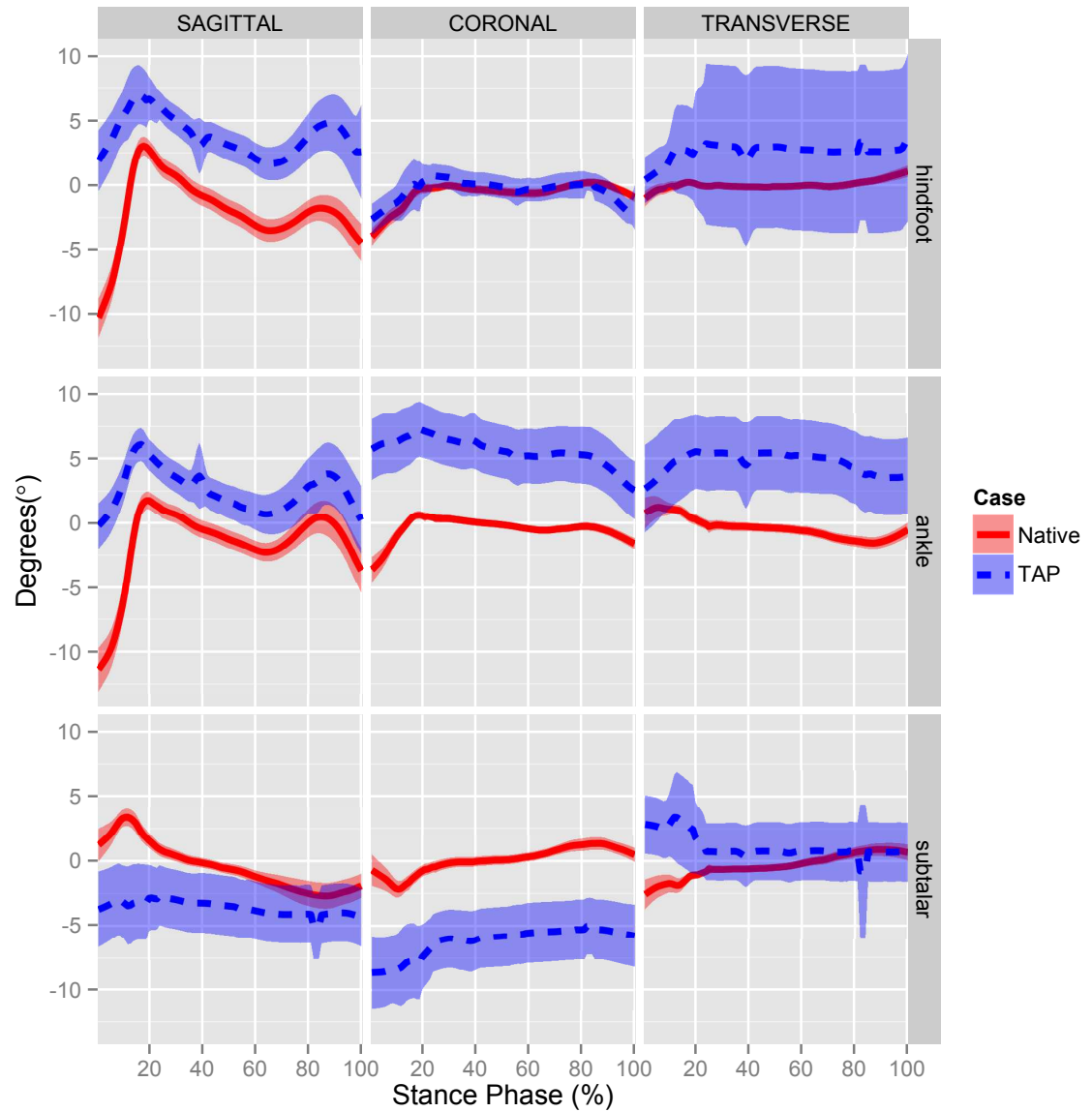

Figure 2: The measured joint kinematics during gait simulations. Each column represents an anatomical plane and each row a joint. The native (red) and TAP (blue) kinematics for all the specimens are presented for the duration of stance phase. 


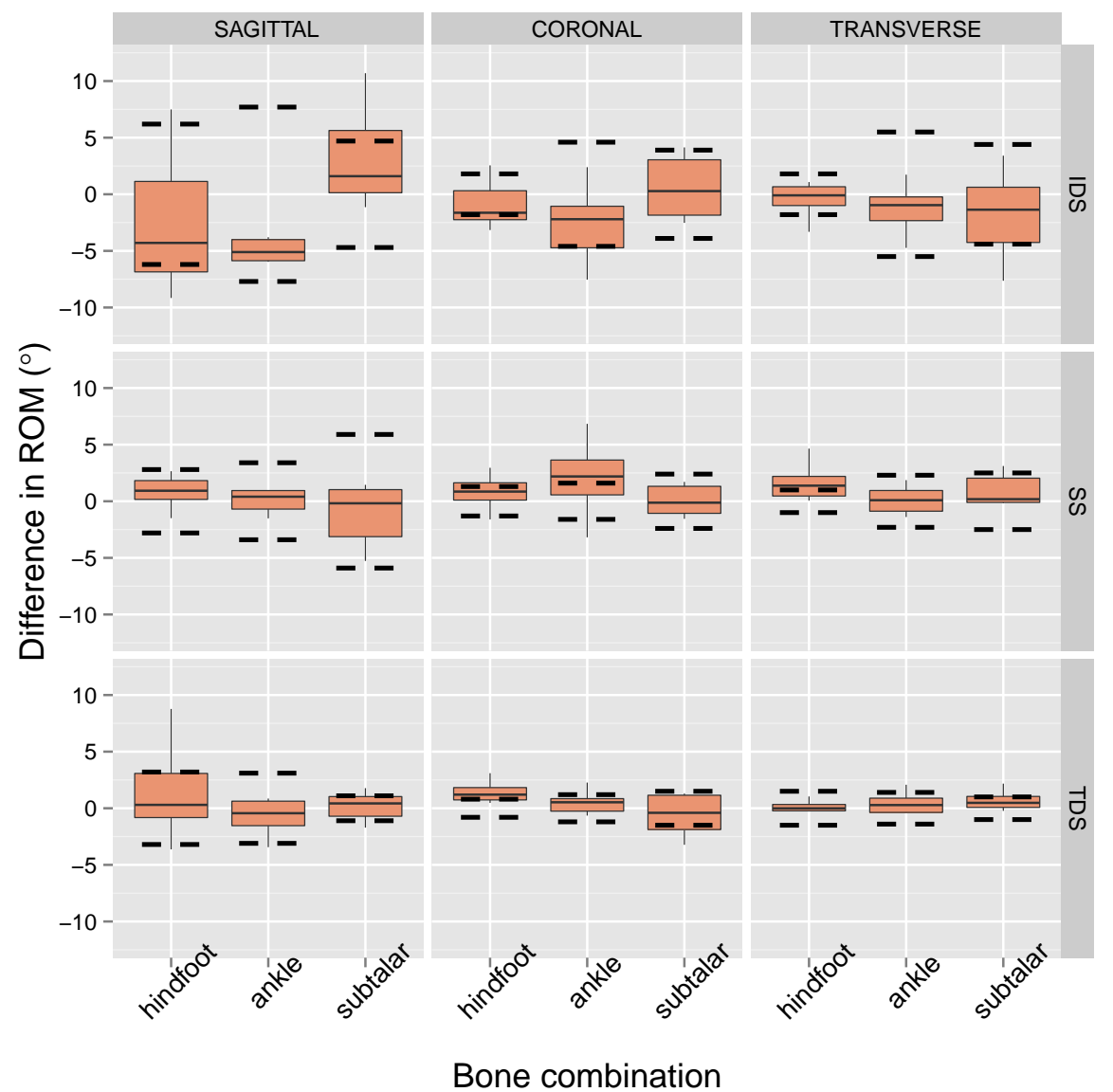

Figure 3: Differences in degrees of range of motion (ROM) for the hindfoot, ankle and subtalar joint for each anatomical plane (Sagittal, Coronal, Transverse) and part of stance (Initial Double Support (IDS), Single Support (SS), Terminal Double Support (TDS)). The width of each box represents the interquartile range, whereas the bottom and top end of the whiskers represent the lowest and highest value still within 1.5 of interquartile ranges respectively. The horizontal line inside the box represents the median. The horizontal dotted lines represent the one standard deviation of the ROM in all the measurements before the implantation. 